\title{
LA ORGANIZACIÓN DOCUMENTAL EN LOS ARCHIVOS DE LA UNIVERSIDAD NACIONAL JORGE BASADRE GROHMANN
}

Responsable: Ing. Eco. Jesús Olivera Cáceres

\section{RESUMEN}

La presente investigación permitió determinar que existen diferencias significativas entre los promedios obtenidos por los grupos experimental y de control en la prueba de Postest, lo cual se atribuye a la aplicación de la capacitación en el Proceso Archivístico Organización del grupo experimental, con sus tres componentes: Clasificación documental, Ordenación documental y Signatura documental, comprobando su eficacia en la adquisición de habilidades en organización documental en el personal administrativo encargado de los Archivos de Gestión de la Universidad Nacional Jorge Basadre Grohmann

\section{ABSTRACT}

The investigation allowed to determine that significant differences exist among the averages obtained by the experimental groups and of control in the test of Post test, which is attributed to the application of the training in the process archivistico organization of the experimental group, with their three components; documental classification, documental ordination and documental signature; checking their effectiveness in the acquisition of abilities in documental organization in the administrative personnel in charge of the files of administration of the National University "Jorge Basadre Grohmann"

\section{INTRODUCCIÓN}

Esta investigación esta orientada a elevar el nivel de conocimientos sobre los aspectos básicos de la archivística concordantes con la organización documental y mejorar las técnicas necesarias en la organización de documentos en los Archivos de Gestión, aplicando la capacitación, en el Proceso Técnico Archivistico Organización, al personal administrativo.

La iniciativa para la elaboración del presente estudio surge de la observación de que en la Universidad Nacional Jorge Basadre Grohmann no existe un tratamiento en los documentos de gestión archivística, lo cual no permiten contribuir a la mejor organización y gestión de su fondo documental.

Es importante el estudio de los procesos archivisticos, especialmente la organización documental, como elemento básico para desarrollar el resto de procesos archivísticos y para poder manejar adecuadamente la documentación y contribuir al desarrollo institucional y, sobre todo, ponernos al servicio de la educación, la cultura y la investigación.

El trabajo contiene cuatro capitulos. En el primero se presenta el planteamiento del problema; en el segundo, el marco teórico; en el tercero, la metodología considerando el tipo y diseño de investigación como, asimismo, el tratamiento técnico del problema. En el cuarto capítulo se hace la presentación de los resultados, la descripción del trabajo de campo, análisis e interpretación de resultados y la verificación de las hipótesis.

Asimismo, se presentan las conclusiones y sugerencias a las que se arribó, así como también la referencia bibliográfica y anexos. En este último se presenta la matriz de consistencia, la operacionalización de la variable y el instrumento para la recolección de datos.

Finalmente, se pretende que la investigación efectuada contribuya a mejorar las habilidades para organizar los documento en el personal administrativo encargado de los Archivos de Gestión de la Universidad Nacional Jorge Basadre Grohmann de Tacna, mediante la Capacitación en el Proceso Técnico Archivistico Organización.

\section{PLANTEAMIENTO DEL PROBLEMA}

\section{Descripción del Problema}

La teoría archivística nos señala que el archivo es el fiel reflejo de la institución productora de documentos. Las secciones 
archivisticas son demostrativas de la organización y de las funciones de la entidad, su evolución y cambios de competencia. Las series corresponden a actividades concretas. La selección se efectúa en forma racional y está avalada por tablas de retención, producto de un estudio completo y consciente de la tipologia documental y sus valores. Una descripción acorde con esta etapa del ciclo vital de los documentos permite la rápida recuperación de la información. Ahora bien, muy pocas veces vemos en los archivos administrativos que la realidad se ajusta a la teoría.

Actualmente en la Universidad Nacional Jorge Basadre Grohmann no existe una adecuada gestión documentaria que oriente el modus operandi y busque la uniformidad del trabajo archivístico universitario. En consecuencia, el Archivo Central, ante la necesidad de salvaguardar el patrimonio, se ve obligado a recibir material sin clasificar o deficientemente clasificado, sin ordenar y con precaria o ninguna descripción. El material no está en condiciones de ser consultado. No conserva su integridad. No puede hablarse de una explotación total del fondo. Ésta es una de las principales causas que impiden el acceso a la consulta. La accesibilidad a los documentos archivisticos exige como condición básica la organización de los fondos documentales.

Este trabajo se realizó en la Universidad Nacional Jorge Basadre Grohmann, en los diferentes archivos de gestión y el archivo de la Universidad, ubicado en la Av. Miraflores y laAv. Cuzco, del departamento, provincia y distrito de Tacna.

\section{Justificación e Importancia}

Teniendo en cuenta que la organización y administración de archivos es un conjunto de principios, métodos y procedimientos orientados a lograr una eficiente organización y funcionamiento de archivos, es función de la archivística la gestión de documentos desde su elaboración hasta la eliminación o conservación permanente de acuerdo con su ciclo vital.

El conocimiento de la legislación archivistica y la capacitación en organización documental nos servirá de mucha utilidad para poder realizar una buena organización y administración de los documentos en los archivos de gestión y el Archivo Central de la Universidad. Nacional Jorge Basadre Grohmann, mejorando el control, conservación, accesibilidad y el servicio de los documentos de archivo. Los archivos son la memoria de las instituciones.

\section{Implementación del Programa de Capacitación}

El proyecto de capacitación cuenta con el apoyo de Secretaria General de la Universidad y los capacitadores serán los encargados del Archivo Central de la Universidad Nacional Jorge Basadre Grohmann de Tacna.

\section{Evaluación del Programa de Capacitación}

La evaluación del proyecto de capacitación se hará mediante el alcance del aprendizaje, administrando pruebas para determinar lo que han aprendido los participantes en el Curso Taller. El procedimiento de evaluación que se utilizará es el diseño de grupos de control antes y después de la prueba. En este procedimiento, se utiliza la misma prueba antes y después de la capacitación. También participa un grupo de control (que no recibe la capacitación) y un grupo experimental (que si la recibe).

Para efectos de la investigación, en cuanto a las puntuaciones en relación con los niveles de habilidades, se asumirá la siguiente evaluación:

La obtención de 0-16 puntos se asumirá como habilidad insatisfactoria, de 16-24 como habilidad básica, asimismo de 24-32 puntos como habilidad competente y finalmente de de 32-40 como habilidad destacada.

\section{MARCO METODOLOGICO}

\section{Tipo de la Investigación}

De acuerdo con la natualeza del estudio que se ha formulado, reúne las condiciones metodológicas suficientes para ser considerada como una investigación APLICADA; y cuyo propósito fundamental es dar solución a problemas prácticos en razón de que en el desarrollo de trabajo se utilizan los procesos archivísticos y la gestión documentaria.

La investigación por su nivel es de CAUSALIDAD, porque nos permitirá mejorar las técnicas necesarias en la organización de documentos en los archivos de gestión, aplicando la capacitación en el Proceso Técnico 
Archivístico Organización, al personal Administrativo que labora en los archivos de gestión de la Universidad Nacional Jorge Basadre Grohmann.

\section{Diseño de la Investigación}

El diseño seleccionado en el presente estudio es CUASI EXPERIMENTAL. En este procedimiento, se utiliza la misma prueba antes (pretest) y después de la capacitación (postest). También participa un grupo de control (que no recibe la capacitación) y un grupo experimental (que sí la recibe). Se asignan en forma aleatoria los capacitandos a cada grupo. Se atribuyen las diferencias de resultados de la prueba anterior y la prueba posterior entre los grupos, a la capacitación que se ha proporcionado.

\section{Diseño Cuasi Experimental}

\begin{tabular}{|l|l|l|l|}
\hline \multicolumn{4}{|c|}{ DISEÑO CON PRE Y POSTEST, CON UN GRUPO DE } \\
CONTROL
\end{tabular}

Donde: GE :Grupo Experimental 01:Prueba pretest

GC:Grupo de control

O2:Prueba postest

\section{Población y Muestra}

La población que abarca la investigación a nivel de los archivos de gestión, académicos y Archivo Central de la Universidad Nacional Jorge Basadre Grohmann está conformada por secretarias, técnicos y profesionales en archivo distribuidos de la siguiente manera:

- Secretarias de Facultad

- Secretarias de Oficina y/o Unidades :32

- Personal de STD - ArchivoM :05

Total General

Considerando la cantidad de la población en cada grupo de informantes, que en un $100 \%$ constituyen 82 administrativos.

La muestra corresponde a 32 administrativos encargados de los archivos de gestión, que representan el $39 \%$ de la población.

El grupo control está constituida por 16 administrativos encargados de los archivos de gestión, asimismo nuestra unidad de análisis está constituida por 16 administrativos encargados de los archivos de gestión, a los cuales se les aplicó la variable independiente como es la Capacitación en el Proceso Técnico Archivistico Organización.

\section{Técnicas e instrumentos para la recolección de datos}

Técnica : Examen

Instrumento: Prueba de entrada (pretest) y Prueba de salida (postest).

5. Técnicas e instrumentos para el procesamiento y representacion de información

En el procesamiento de los datos se utilizan tablas de distribución de frecuencias, medidas de tendencia central como la media aritmética y de dispersión como la desviación estándar.

La media aritmética es la nota promedio a nivel de grupo. La dispersión estándar indica si un grupo es homogéneo o heterogéneo.La distribución de frecuencias se simplifica en gráficos de barras, los cuales se realizan teniendo en cuenta los intervalos de frecuencia.

\section{Técnicas e instrumentos para el análisis e interpretación de datos}

Para el análisis e interpretación de datos, se utiliza un plan de análisis estadístico inferencial, con la aplicación de la prueba de T de Student, como prueba de significación con un margen de error 0,5\%.La interpretación se realiza mediante la verificación de la hipótesis de investigación.

\section{CONCLUSIONES}

1. El nivel de habilidades en el personal administrativo encargado de los archivos de gestión, en el grupo experimental y en el de control, antes de aplicar la capacitación en el Proceso Técnico Archivístico Organización, demuestra que no existen diferencias significativas entre los promedios obtenidos mediante el estadístico T de student.

2. Se aplicó la capacitación en el Proceso Técnico Archivistico Organización al personal administrativo encargado de los archivos de gestión,con sus tres componentes: Clasificación documental, Ordenación documental y Signatura documental, demostrando su eficacia en la adquisición de habilidades en organización documental con un $5 \%$ de nivel de significación.

3. Existen diferencias significativas en el nivel de habilidades del personal administrativo 
encargado de los archivos de gestión en el grupo experimental y de control después de aplicar la capacitación en el Proceso Técnico Archivistico Organización, lo cual se demuestra en los promedios obtenidos mediante el estadistico T de student.

\section{RECOMENDACIONES}

Promover la capacitación en el Proceso Técnico Archivistico Organización como un medio para mejorar las habilidades en organización documental en todo el personal administrativo encargado de los archivos de gestión, ya sean estos archivos de gestión académicos o administrativos.

Continuar con investigaciones orientadas a elaborar y validar documentos que permitan mejorar u optimizar el trabajo técnico en organización documental en un archivo de gestión como el Cuadro de clasificación de documentos, Libros de registro de documentos y el Manual de gestión de documentos.

Que las instituciones de formación archivistica, prioricen la formación y capacitación en procesos archivísticos, especialmente la organización documental, como elemento básico para desarrollar el resto de procesos archivisticos y para poder manejar adecuadamente la documentación y contribuir al desarrollo institucional $y$, sobre todo, ponernos al servicio de la educación, la cultura y la investigación.

\section{BIBLIOGRAFIA}

Cárdenas Ayaipoma, Mario. Manual de organización de documentos archivisticos. Fondo Pro Archivo, Grupo Esperanza del Perú, Lima, 2004.

Conde Villaverde, Maria Luisa. Manual de tratamiento de archivos administrativos. Dirección de Archivos Estatales, Ministerio de Educación de España, Madrid, 1992.

Escuela Nacional de Archiveros.Curso Básico deArchivos, Lima, 2005.

Cruz Mondet, José Ramón. Manual de Archivistica, Madrid, 1994.

Cruz Mondet, José Ramón. "La gestión de documentos y archivos: una herramienta al servicio de la calidad", Revista -D'Arxius, Associació D'Arxivers Valencians, Madrid, 2002.

Fung Henriquez, Vilma, Lo serio de las series. PUCP, Lima, 2003.

Gutiérrez Muñoz, César. Archivistica. Lima, 1991.

Kuon Carrero, Rosa. Manual de los archivos universitarios. Biblioteca Nacional del Perú, Lima. 1999.

Paloma Fernández, Gil. Manual de organización de archivos de gestión en las oficinas municipales. Ediciones Adhara, Granada,1997. 